\title{
An efficient scattering model for PEC and penetrable nanowires on a dielectric substrate
}

\author{
M. Karamehmedović \\ mirza@iwt.uni-bremen.de \\ P.-E. Hansen \\ peh@dfm.dtu.dk
}

T. Wriedt

thw@iwt.uni-bremen.de

\begin{abstract}
Dept. of Process and Chemical Engineering, University of Bremen, Badgasteiner Str. 3, 28359 Bremen, Germany

Danish Fundamental Metrology, Technical University of Denmark, Matematiktorvet 307, DK-2800 Kgs. Lyngby, Denmark

Dept. of Process and Chemical Engineering, University of Bremen, Badgasteiner Str. 3, 28359 Bremen, Germany
\end{abstract}

An efficient forward scattering model, based on the Method of Auxiliary Sources, is formulated for perfectly electrically conducting (PEC) and penetrable nanowires on dielectric substrates. The accuracy of the model is investigated parametrically, with emphasis on future application in an inverse scattering scheme. The model is tested on families of PEC and silver nanowires on silicon substrate. [DOI: 10.2971/jeos.2011.11021]

Keywords: particle on surface scattering, method of auxiliary sources

\section{INTRODUCTION}

Production and quality control of nanoscale structures in materials typically rely on nondestructive measurement techniques, where the problem is to reconstruct or estimate parameters of the sample under investigation from the measured optical power in the scattered far field. The sought parameters are, e.g., the size, shape and material composition of a nanoparticle on a substrate, and the measurement technique is, e.g., Optical Diffraction Microscopy (ODM) [1]-[4]. In an industrial context, the reconstruction of the parameters (or inversion) needs to be rapid and robust. It can be done using a table-based approach, as described in Karamehmedović et al. [5]. When the use of look-up tables is undesirable or impractical, for example due to high dimensionality of the inverse problem, a common approach is some variant of the Kirsch-Kress Method (KKM) [6], [7, Section 7.3]. Here, essentially, a forward scattering model is used to reconstruct the near field from the measured far field, and the shape of the nanoparticle is estimated by minimising the boundary condition error over a class of admissible particle surfaces. Since this minimisation typically requires many evaluations of the near field, the efficiency of the forward model greatly influences the overall efficiency of the inversion.

Apart from the major issues as ill-posedness of the inverse problem and noise, which are outside the scope of this paper, obtaining a fast KKM-type method of characterisation of nanoparticles on substrates may be difficult due to two outstanding problems: first, since the measured structure is electrically small, it may be necessary to use a full Maxwell model, rather than asymptotic formulations, to achieve the desired accuracy of inversion. Second, since the measured structure is placed on a dielectric substrate, the resulting scattered field is described by radiation integrals that involve a half-space (or half-plane) Green's function. Evaluation of such Green's functions, in turn, involves Sommerfeld-type integrals, and is therefore numerically expensive. For particles with relatively simple morphology, the far-field scattering data alone may suffice for characterisation, and in this case an efficient, asymptotic approximation of the Sommerfeld integrals can be used. In general, however, reconstruction of the near field is needed.

The Method of Auxiliary Sources (MAS) [8]-[14] is an efficient non-asymptotic scheme for numerical solution of boundary problems. It is relatively easy to implement, and it can outperform the traditional integral equation-based methods. In [5], we demonstrated that the MAS can be used to construct an efficient forward scattering model for a table-based measurement of non-periodic defects in nanogratings based only on given far-field amplitude data. ${ }^{1}$ Furthermore, the MAS was used in conjunction with the Kirsch-Kress Method by Tal and Leviatan [15], Lin and Kiang [16], and Obelleiro et al. [17] to reconstruct perfectly electrically conducting cylinders in twodimensional free space (with both phase and amplitude of the scattered field given). More recently, Alves and Martins [18], reconstructed the shape and location of inclusions and cavities in elastic bodies (with all Cauchy data given) using a MASKKM approach.

In this paper we construct and numerically investigate a MAS formulation of scattering by perfectly electrically conducting (PEC) or penetrable nanowires on dielectric substrates. A formulation of the same type as ours was already reported by

\footnotetext{
${ }^{1}$ It has come to our attention that the complex refractive index of the nanograting material used in the numerical example in [5, Section 5] corresponds to an active nanograting, rather than a silicon nanograting.
} 
Eremina et al. [19, 20] for three-dimensional scattering by axially symmetric, nonspherical, penetrable nanoparticles on plane surfaces. However, since we intend to use our formulation in a KKM-type inversion in a future publication, we here emphasise efficiency and approximate the Sommerfeld-type integrals that occur in the half-plane Green's function using simple Fresnel reflection. Our main purpose here is, given a concrete type of scattering problem, to establish good parameter values for such simplified MAS forward model, and to investigate its accuracy, efficiency and range. In particular, we consider scattering by PEC and silver $(\mathrm{Ag})$ nanowires of circular cross section and placed on a semi-infinite silicon (Si) substrate. The illumination is time-harmonic with free-space wavelength $\lambda_{0}=325 \mathrm{~nm}$, and the nanowire cross sections have radii from $0.2 \lambda_{0}$ to $1.2 \lambda_{0}$. Both far-field and near-field results are presented, and the errors are investigated parametrically. The commercially available FEM software package COMSOL® Multiphysics $[21,22]$ is used to produce reference results. It is stressed that we here do not intend any formal comparison of the computation times of our forward model and COMSOL.

In Section 2, the MAS-based scattering model is described. Numerical results are presented in Section 3. Conclusions and suggestions for future work are given in Section 4 .

\section{THE SCATTERING MODEL}

The scattering model is here described using a concrete twodimensional scattering problem. The geometry of the problem is outlined in Figure 1. A PEC or silver (Ag) nanowire is positioned on a planar, semi-infinite silicon ( $\mathrm{Si}$ ) substrate. It is assumed that the length of the nanowire is much greater than the characteristic size of its cross section, and that a twodimensional model is sufficiently accurate. The nanowire is illuminated by a time-harmonic uniform plane wave of unit amplitude and incident in the normal direction on the substrate. The illumination is transverse-electric (TE) polarised, that is, the incident electric field vector is perpendicular to the plane of the nanowire cross section. The time dependence factor $e^{j \omega t}$ is in the following suppressed for simplicity. The operating free-space wavelength $\lambda_{0}$ is $325 \mathrm{~nm}$. According to Palik [23], the corresponding complex refractive indices of silicon and silver are $n_{S i}=5.05506-j 3.20418$ and $n_{A g}=0.571-j 0.636$, respectively. Figure 2 shows the introduced Cartesian coordinate system (with origin $O_{1}$ at the point of contact between nanowire and substrate) and cylindrical coordinate system (with origin $\mathrm{O}_{2}$ at the center of the cylinder cross section).

Note the unit vectors $\widehat{\mathbf{x}}, \widehat{\mathbf{y}}, \widehat{\mathbf{z}}, \widehat{\rho}$ and $\widehat{\phi}$ pointing in the respective coordinate directions. The cylinder cross section is represented by a closed disk $\Sigma$ with (circular) boundary $\sigma$ of radius $r$. The air-silicon interface is represented by the line $\gamma$. The incident field propagates in the negative $y$ direction, and it is given by $\left(\mathbf{E}^{i}, \mathbf{H}^{i}\right)=\left(\widehat{\mathbf{z}} e^{j k_{0} y},-\widehat{\mathbf{x}} \eta_{0}^{-1} e^{j k_{0} y}\right)$, where $k_{0}=2 \pi / \lambda_{0}$ is its wavenumber and $\eta_{0} \approx 377 \Omega$ is the free-space impedance. In the absence of the nanowire, the incident field would be reflected and refracted at the airsilicon interface, giving rise to a reflected uniform plane wave

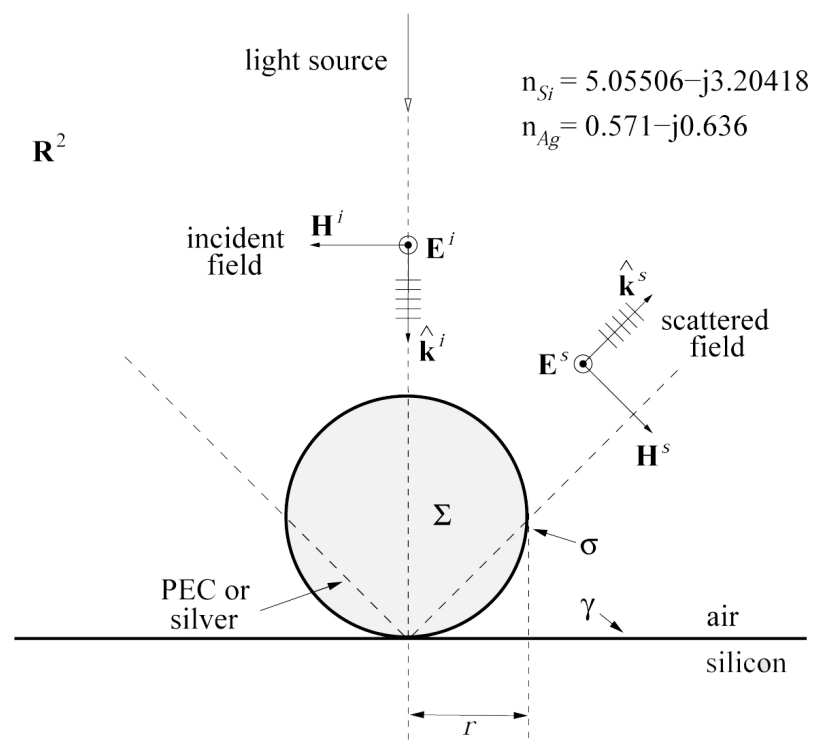

FIG. 1 A nanowire on a silicon substrate - a model of the measurement setup (cross section).

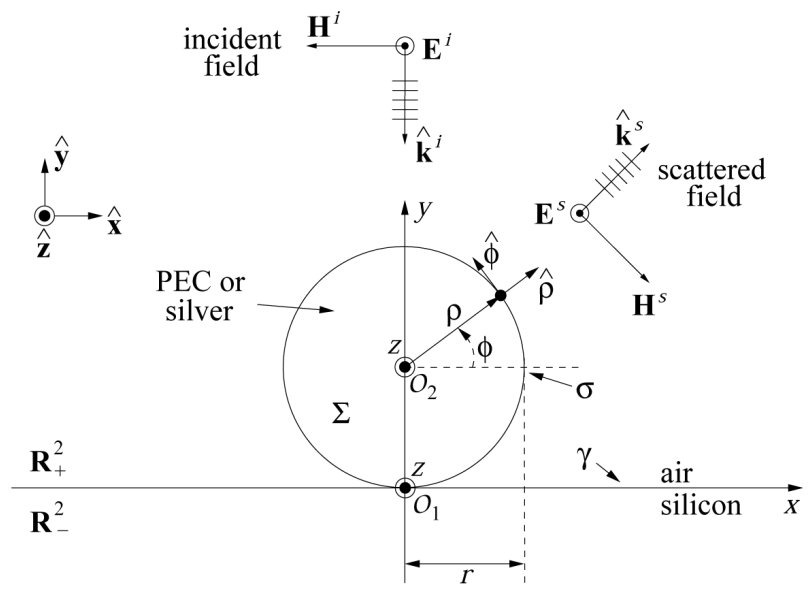

FIG. 2 The introduced Cartesian and cylindrical coordinate systems.

$\left(\mathbf{E}^{r e f l}, \mathbf{H}^{r e f l}\right)=\Gamma_{r}\left(\widehat{\mathbf{z}} e^{-j k_{0} y}, \widehat{\mathbf{x}} \eta_{0}^{-1} e^{-j k_{0} y}\right)$ in the upper half-plane $\mathbf{R}_{+}^{2}$ propagating in the positive $y$ direction, as well as a transmitted uniform plane wave

$$
\left(\mathbf{E}^{\text {trans }}, \mathbf{H}^{\text {trans }}\right)=\Gamma_{t}\left(\widehat{\mathbf{z}} e^{j k_{A g} y},-\widehat{\mathbf{x}} n_{A g} \eta_{0}^{-1} e^{j k_{A g} y}\right)
$$

in the lower half-plane $\mathbf{R}_{-}^{2}$ propagating in the negative $y$ direction. Here, $k_{A g}=k_{0} n_{A g} / n_{0}$, and the Fresnel reflection coefficient $\Gamma_{r}$ and transmission coefficient $\Gamma_{t}$ for the air-silicon interface at $325 \mathrm{~nm}$ are given by

$$
\begin{aligned}
\Gamma_{r} & =\frac{n_{0}-n_{S i}}{n_{0}+n_{S i}} \approx-0.742+j 0.137, \\
\Gamma_{t} & =\frac{2 n_{0}}{n_{0}+n_{S i}} \approx 0.258+j 0.137,
\end{aligned}
$$

where $n_{0}=1$ is the refractive index of air. The fields $\left(\mathbf{E}^{i}+\mathbf{E}^{r e f l}, \mathbf{H}^{i}+\mathbf{H}^{\text {refl }}\right)$ and $\left(\mathbf{E}^{\text {trans }}, \mathbf{H}^{\text {trans }}\right)$ satisfy the transmission conditions at the boundary $\gamma$. In the following, for the scattering problem with the nanowire present, we write the total field $\left(\mathbf{E}^{t o t}, \mathbf{H}^{\text {tot }}\right)$ in the upper half-plane as the sum of the incident field $\left(\mathbf{E}^{i}, \mathbf{H}^{i}\right)$, the reflected field $\left(\mathbf{E}^{r e f l}, \mathbf{H}^{r e f l}\right)$ and some unknown scattered field $\left(\mathbf{E}^{s}, \mathbf{H}^{s}\right)$. In the lower half-plane, the total field is written as the sum of the transmitted field ( $\left.\mathbf{E}^{\text {trans }}, \mathbf{H}^{\text {trans }}\right)$ and an unknown scattered field 
$\left(\mathbf{E}^{s}, \mathbf{H}^{s}\right)$. We wish to find the scattered field $\left(\mathbf{E}^{s}, \mathbf{H}^{s}\right)$ in the upper half-plane. In the PEC case, the total tangential electric field vanishes at the nanowire cross section boundary $\sigma$. In the penetrable case (Ag nanowire), the total tangential electric and magnetic fields are continuous across the boundary $\sigma$. In both cases, the total tangential electric and magnetic fields are continuous across the air-silicon interface $\gamma$. In the following, subscripts $\sigma+$ and $\sigma$ - mean limit values at $\sigma$ taken from the exterior $\mathbf{R}_{+}^{2} \backslash \Sigma$ and from the interior $\Sigma^{\circ}$ of the nanowire cross section, respectively. Subscripts $\gamma+$ and $\gamma$ - indicate limit values at $\gamma$ taken from the upper half-plane $\mathbf{R}_{+}^{2}$ and the lower half-plane $\mathbf{R}_{-}^{2}$, respectively. Thus

$$
\begin{aligned}
\widehat{\rho} \times \mathbf{E}_{\sigma+}^{s}= & -\widehat{\rho} \times\left(\mathbf{E}_{\sigma_{+}}^{i}+\mathbf{E}_{\sigma_{+}}^{r e f l}\right) \quad \text { on } \sigma, \\
\widehat{\mathbf{y}} \times\left(\mathbf{E}_{\gamma_{+}}^{s}-\mathbf{E}_{\gamma_{-}}^{s}\right) & =-\widehat{\mathbf{y}} \times\left(\mathbf{E}_{\gamma_{+}}^{i}+\mathbf{E}_{\gamma_{+}}^{r e f l}-\mathbf{E}_{\gamma_{-}}^{\text {trans }}\right) \\
& =0 \text { on } \gamma, \\
\widehat{\mathbf{y}} \times\left(\mathbf{H}_{\gamma_{+}}^{s}-\mathbf{H}_{\gamma_{-}}^{s}\right) & =-\widehat{\mathbf{y}} \times\left(\mathbf{H}_{\gamma_{+}}^{i}+\mathbf{H}_{\gamma_{+}}^{\text {refl }}-\mathbf{H}_{\gamma_{-}}^{\text {trans }}\right) \\
& =0 \text { on } \gamma
\end{aligned}
$$

in the PEC case, and

$$
\begin{aligned}
\widehat{\rho} \times\left(\mathbf{E}_{\sigma+}^{s}-\mathbf{E}_{\sigma-}^{t o t}\right) & =-\widehat{\rho} \times\left(\mathbf{E}_{\sigma+}^{i}+\mathbf{E}_{\sigma+}^{r e f l}\right) \quad \text { on } \sigma, \\
\hat{\rho} \times\left(\mathbf{H}_{\sigma+}^{s}-\mathbf{H}_{\sigma-}^{t o t}\right) & =-\widehat{\rho} \times\left(\mathbf{H}_{\sigma+}^{i}+\mathbf{H}_{\sigma+}^{r e f l}\right) \quad \text { on } \sigma, \\
\widehat{\mathbf{y}} \times\left(\mathbf{E}_{\gamma_{+}}^{s}-\mathbf{E}_{\gamma_{-}}^{s}\right) & =-\widehat{\mathbf{y}} \times\left(\mathbf{E}_{\gamma_{+}}^{i}+\mathbf{E}_{\gamma_{+}}^{r e f l}-\mathbf{E}_{\gamma_{-}}^{\text {trans }}\right) \\
& =0 \quad \text { on } \gamma, \\
\widehat{\mathbf{y}} \times\left(\mathbf{H}_{\gamma_{+}}^{s}-\mathbf{H}_{\gamma_{-}}^{s}\right) & =-\widehat{\mathbf{y}} \times\left(\mathbf{H}_{\gamma_{+}}^{i}+\mathbf{H}_{\gamma_{+}}^{\text {refl }}-\mathbf{H}_{\gamma_{-}}^{\text {trans }}\right) \\
& =0 \quad \text { on } \gamma
\end{aligned}
$$

in the penetrable case.

Figures 3 and 4 show the employed MAS formulation of the scattering problem. The scatterer is replaced by a number, say $N^{\prime}$, of so-called auxiliary sources located inside the crosssection domain $\Sigma$ at a number of selected points $\mathbf{x}_{1}^{\prime}, \ldots, \mathbf{x}_{N^{\prime}}^{\prime}$ and with (unknown) complex amplitudes $C_{1}, \ldots, C_{N^{\prime}}$. The auxiliary sources are $z$-directed electric line currents radiating in the presence of the silicon substrate. Thus, in the exterior of the cylinder cross section in the upper half-plane, the scattered field $\left(\mathbf{E}^{S}, \mathbf{H}^{S}\right)$ is approximated by the finite linear combinations

$$
\begin{gathered}
\mathbf{E}^{S}(\mathbf{x}) \approx \widehat{\mathbf{z}} \sum_{v=1}^{N^{\prime}} C_{v} \Phi_{1 / 2}\left(\mathbf{x}, \mathbf{x}_{v}^{\prime}\right), \quad \mathbf{x} \in \mathbf{R}_{+}^{2} \backslash \Sigma, \\
\mathbf{H}^{S}(\mathbf{x}) \approx \frac{j}{\omega \mu_{0}} \sum_{v=1}^{N^{\prime}} C_{v}\left(-\widehat{\mathbf{y}} \partial_{x} \Phi_{1 / 2}\left(\mathbf{x}, \mathbf{x}_{v}^{\prime}\right)\right. \\
\left.+\widehat{\mathbf{x}} \partial_{y} \Phi_{1 / 2}\left(\mathbf{x}, \mathbf{x}_{v}^{\prime}\right)\right), \quad \mathbf{x} \in \mathbf{R}_{+}^{2} \backslash \Sigma,
\end{gathered}
$$

where $\Phi_{1 / 2}\left(\cdot, \mathbf{x}_{v}^{\prime}\right)$ is the outgoing fundamental solution of the two-dimensional Helmholtz operator in the air-silicon medium, with singularity at $\mathbf{x}_{v}^{\prime}$. In the upper half-plane $\mathbf{R}_{+}^{2}$,

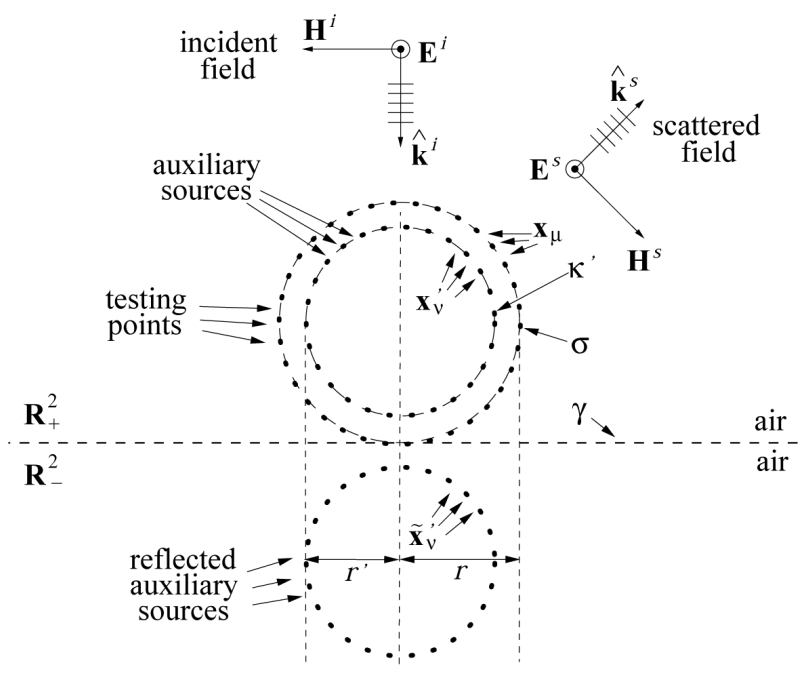

FIG. 3 The MAS formulation of the inverse problem: reconstructing the scattered field outside the cylinder cross section.

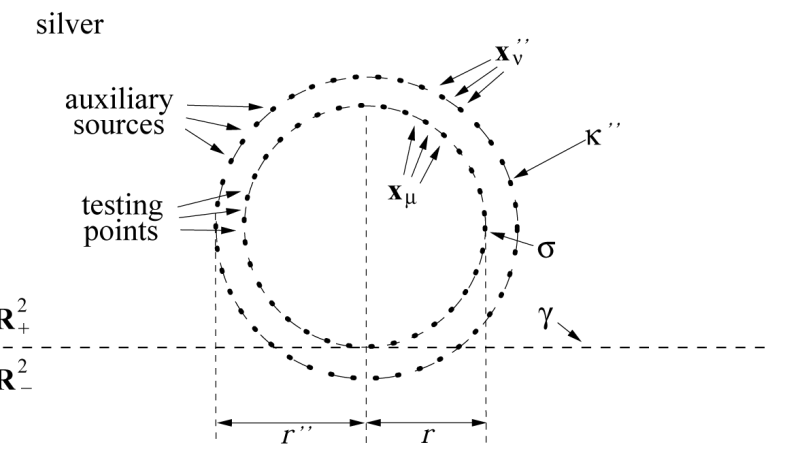

FIG. 4 The MAS formulation of the inverse problem: reconstructing the total field inside the cross section of a penetrable cylinder.

we may approximate the function $\Phi_{1 / 2}$ by the computationally relatively cheap function

$$
\begin{aligned}
\Phi_{1 / 2}\left(\mathbf{x}, \mathbf{x}^{\prime}\right) \approx & H_{0}^{(2)}\left(k_{0}\left|\mathbf{x}-\mathbf{x}^{\prime}\right|\right) \\
& +\Gamma_{r} H_{0}^{(2)}\left(k_{0}\left|\mathbf{x}-\widetilde{\mathbf{x}}^{\prime}\right|\right), \quad \mathbf{x}, \mathbf{x}^{\prime} \in \mathbf{R}_{+}^{2},
\end{aligned}
$$

where $H_{0}^{(2)}$ is the Hankel function of zero order and of the second kind, and where $\widetilde{\mathbf{x}}^{\prime}=\left(x^{\prime},-y^{\prime}\right)$ is the reflection of the point $\mathbf{x}^{\prime}=\left(x^{\prime}, y^{\prime}\right)$ with respect to the $x$-axis. This gives the following simplified approximation of the scattered field in $\mathbf{R}_{+}^{2} \backslash \Sigma$ :

$$
\begin{aligned}
\mathbf{E}^{\mathcal{S}}(\mathbf{x}) \approx \widehat{\mathbf{z}} \sum_{v=1}^{N^{\prime}} C_{v}\left(H_{0}^{(2)}\left(k_{0}\left|\mathbf{x}-\mathbf{x}_{v}^{\prime}\right|\right)\right. & \left.+\Gamma_{r} H_{0}^{(2)}\left(k_{0}\left|\mathbf{x}-\widetilde{\mathbf{x}}_{v}^{\prime}\right|\right)\right) \\
\mathbf{H}^{\mathcal{S}}(\mathbf{x}) \approx & \frac{j}{\eta_{0}} \sum_{v=1}^{N^{\prime}} C_{v}\left|\mathbf{x}-\mathbf{x}_{v}^{\prime}\right|^{-1}\left(\left(-\widehat{\mathbf{x}}\left(y-y_{v}^{\prime}\right)\right.\right. \\
& \left.+\widehat{\mathbf{y}}\left(x-x_{v}^{\prime}\right)\right) H_{1}^{(2)}\left(k_{0}\left|\mathbf{x}-\mathbf{x}_{v}^{\prime}\right|\right) \\
& +\Gamma_{r}\left(-\widehat{\mathbf{x}}\left(y+y_{v}^{\prime}\right)+\widehat{\mathbf{y}}\left(x-x_{v}^{\prime}\right)\right) \\
& \left.\times H_{1}^{(2)}\left(k_{0}\left|\mathbf{x}-\widetilde{\mathbf{x}}_{v}^{\prime}\right|\right)\right) .
\end{aligned}
$$

In the simplified approximation, the scattered field in $\mathbf{R}_{+}^{2} \backslash \Sigma$ is radiated by a set of $z$-directed electrical line currents placed 
at $\mathbf{x}_{v}^{\prime}$ and with amplitudes $C_{v}$, together with their 'reflections in the silicon substrate', that is, z-directed electrical line currents placed at $\widetilde{\mathbf{x}}_{v}^{\prime}$ and with amplitudes $\Gamma_{r} C_{v}$ (see Figure 3 ). Both the original and the reflected line currents radiate in free space.

In the PEC case, the total field $\left(\mathbf{E}^{\text {tot }}, \mathbf{H}^{\text {tot }}\right)$ in the interior of the nanowire cross section $\Sigma$ is identically zero. In the penetrable case, this field is approximated by the finite linear combinations

$$
\begin{aligned}
& \mathbf{E}^{t o t}(\mathbf{x}) \approx \widehat{\mathbf{z}} \sum_{v=1}^{N^{\prime \prime}} D_{v} H_{0}^{(2)}\left(k_{A g}\left|\mathbf{x}-\mathbf{x}_{v}^{\prime \prime}\right|\right), \quad \mathbf{x} \in \Sigma^{\circ}, \\
& \mathbf{H}^{t o t}(\mathbf{x}) \approx \frac{j \eta_{A g}}{\eta_{0}} \sum_{v=1}^{N^{\prime \prime}} D_{v}\left|\mathbf{x}-\mathbf{x}_{v}^{\prime \prime}\right|^{-1}\left(-\widehat{\mathbf{x}}\left(y-y_{v}^{\prime \prime}\right)\right. \\
&\left.+\widehat{\mathbf{y}}\left(x-x_{v}^{\prime \prime}\right)\right) H_{1}^{(2)}\left(k_{A g}\left|\mathbf{x}-\mathbf{x}_{v}^{\prime \prime}\right|\right), \quad \mathbf{x} \in \Sigma^{\circ},
\end{aligned}
$$

of fields produced by auxiliary sources located at selected points $x_{1}^{\prime \prime}, \ldots, x_{N^{\prime \prime}}^{\prime \prime}$ in the exterior of the nanowire cross section (see Figure 4), and with (unknown) complex amplitudes $D_{1}, \ldots, D_{N^{\prime \prime}}$. The Hankel function $H_{0}^{(2)}$ is the outgoing fundamental solution of the two-dimensional Helmholtz operator. These auxiliary sources radiate in the silver-filled plane.

Inserting the expansion (13) into the boundary condition (3), and requiring that (3) be fulfilled at some chosen testing points $\mathbf{x}_{1}, \ldots, \mathbf{x}_{M}$ at the boundary $\sigma$, we obtain the following system of linear equations in the unknown auxiliary source amplitudes $C_{v}$ for the PEC case:

$$
\begin{gathered}
\sum_{v=1}^{N^{\prime}} C_{v}\left(H_{0}^{(2)}\left(k_{0}\left|\mathbf{x}_{\mu}-\mathbf{x}_{v}^{\prime}\right|\right)+\Gamma_{r} H_{0}^{(2)}\left(k_{0}\left|\mathbf{x}_{\mu}-\widetilde{\mathbf{x}}_{v}^{\prime}\right|\right)\right) \\
=-\left(e^{j k_{0} y_{\mu}}+\Gamma_{r} e^{-j k_{0} y_{\mu}}\right), \quad \mu=1, \ldots, M
\end{gathered}
$$

Similarly, inserting the expansions (13)-(16) into the transmission conditions (6) and (7), and requiring that (6)-(7) be fulfilled at the test points $\mathbf{x}_{1}, \ldots \mathbf{x}_{M}$, we obtain the following system for the penetrable case:

$$
\begin{aligned}
\sum_{v=1}^{N^{\prime}} C_{v} & \left(H_{0}^{(2)}\left(k_{0}\left|\mathbf{x}_{\mu}-\mathbf{x}_{v}^{\prime}\right|\right)+\Gamma_{r} H_{0}^{(2)}\left(k_{0}\left|\mathbf{x}_{\mu}-\widetilde{\mathbf{x}}_{v}^{\prime}\right|\right)\right) \\
& -\sum_{v=1}^{N^{\prime \prime}} D_{v} H_{0}^{(2)}\left(k_{A g}\left|\mathbf{x}_{\mu}-\mathbf{x}_{v}^{\prime \prime}\right|\right) \\
= & -\left(e^{j k_{0} y_{\mu}}+\Gamma_{r} e^{-j k_{0} y_{\mu}}\right), \quad \mu=1, \ldots, M
\end{aligned}
$$

$$
\begin{aligned}
\frac{j}{\eta_{0}} \sum_{v=1}^{N^{\prime}} C_{v}\left|\mathbf{x}_{\mu}-\mathbf{x}_{v}^{\prime}\right|^{-1}\left(H _ { 1 } ^ { ( 2 ) } ( k _ { 0 } | \mathbf { x } _ { \mu } - \mathbf { x } _ { v } ^ { \prime } | ) \left(\left(y_{\mu}-y_{v}^{\prime}\right) \sin \phi_{\mu}\right.\right. \\
\left.+\left(x_{\mu}-x_{v}^{\prime}\right) \cos \phi_{\mu}\right)+\Gamma_{r} H_{1}^{(2)}\left(k_{0}\left|\mathbf{x}_{\mu}-\widetilde{\mathbf{x}}_{v}^{\prime}\right|\right) \\
\left.\quad \times\left(\left(y_{\mu}+y_{v}^{\prime}\right) \sin \phi_{\mu}+\left(x_{\mu}-x_{v}^{\prime}\right) \cos \phi_{\mu}\right)\right) \\
\quad-\frac{j n_{A g}}{\eta_{0}} \sum_{v=1}^{N^{\prime \prime}} D_{v}\left|\mathbf{x}_{\mu}-\mathbf{x}_{v}^{\prime \prime}\right|^{-1} H_{1}^{(2)}\left(k_{A g}\left|\mathbf{x}_{\mu}-\mathbf{x}_{v}^{\prime \prime}\right|\right) \\
\quad \times\left(\left(x_{\mu}-x_{v}^{\prime \prime}\right) \cos \phi_{\mu}+\left(y_{\mu}-y_{v}^{\prime \prime}\right) \sin \phi_{\mu}\right) \\
=\frac{\sin \phi_{\mu}}{\eta_{0}}\left(\Gamma_{r} e^{-j k_{0} y_{\mu}}-e^{j k_{0} y_{\mu}}\right), \quad \mu=1, \ldots, M .
\end{aligned}
$$

The angles $\phi_{\mu}$ are the azimuthal coordinates of the testing points $\mathbf{x}_{1}, \ldots, \mathbf{x}_{M}$. A MAS approximation to the scattered field $\left(\mathbf{E}^{s}, \mathbf{H}^{s}\right)$ in $\mathbf{R}_{+}^{2} \backslash \Sigma$ is found by solving (17) for the amplitudes $C_{v}$ (PEC case) or by solving (18)-(19) for the amplitudes $C_{v}$, $D_{v}$ (penetrable case) and inserting the solution into (13)-(14). In the penetrable case, an approximation to the total field in the interior of the nanowire cross section is obtained by inserting the solution $D_{1}, \ldots, D_{N^{\prime \prime}}$ into (15)-(16). Since the fields expressed by the finite linear combinations of the form (13) or (14) are continuous in a neighbourhood of the boundary $\gamma$ in $\mathbf{R}^{2}$, they satisfy the transmission conditions (4)-(5) and (8)-(9).

\section{NUMERICAL RESULTS}

In our concrete implementation, it is always the case that $N^{\prime}=N^{\prime \prime}=M$, and we therefore use the common symbol $N$ for these quantities. The interior auxiliary sources (located at $\mathbf{x}_{1}^{\prime}, \ldots, \mathbf{x}_{N}^{\prime}$ ), the testing points (located at $\mathbf{x}_{1}, \ldots, \mathbf{x}_{N}$ ) and the exterior auxiliary sources (located at $\mathbf{x}_{1}^{\prime \prime}, \ldots, \mathbf{x}_{N}^{\prime \prime}$ ) are uniformly distributed along the circles $\kappa^{\prime}, \sigma$ and $\kappa^{\prime \prime}$, respectively, of radii $0.86 r, r$ and $r / 0.86 \approx 1.16 r$ and concentric with the nanowire cross section $\sigma$. The values $0.86 r$ and $r / 0.86$ for the radii of $\kappa^{\prime}$ and $\kappa^{\prime \prime}$ were found adequate through numerical experimentation. Other values can of course be used, but it should be kept in mind that the condition number of the system matrix for the calculation of the auxiliary source amplitudes can increase significantly when the distance between the auxiliary sources and the testing points is too large. The three point distributions use the same set of angles (azimuthal coordinates $\phi)$; this generally decreases the condition number of the system matrices for the systems (17) and (18)-(19), as compared to the case where the auxiliary sources and the testing points are mutually 'offset.' None of the azimuthal coordinates are $\phi=-90^{\circ}$ or $\phi=90^{\circ}$, so none of the testing points are at the contact point $O_{1}$ between the nanowire and the substrate or at the 'top' of the nanowire cross section. Numerical investigation shows an increase in the far-field error otherwise. Finally, the symmetry of the scattering problem with respect to the $y$-axis is exploited to halve the number of the unknown auxiliary source amplitudes and the number of linear equations used to find these amplitudes. Thus, when we in the following state that $N$ auxiliary sources and testing points are used for a PEC nanowire, the MAS formulation formally employs $2 \mathrm{~N}$ auxiliary sources and $2 N$ testing points (but has only $N$ linear equations for $N$ unknown auxiliary source amplitudes.) Similarly, when we state that $N$ auxiliary sources and testing points are used for a silver nanowire, the MAS formulation formally employs $2 \mathrm{~N}$ sources (with reflections) for the scattered field, $2 \mathrm{~N}$ sources for the interior field (see Figures 3 and 4 ), and $2 \times 2 N$ testing points (but has only $2 N$ linear equations for $2 \mathrm{~N}$ unknown auxiliary source amplitudes.)

To measure the deviation of the MAS far-field results from the reference over some sampling interval $I \subseteq\left[0^{\circ}, 90^{\circ}\right]$, introduce

$$
\varepsilon_{f a r}(I)=\frac{\left\|E_{f a r}^{C O M S O L}-E_{f a r}^{M A S}\right\|_{L^{2}(I)}}{\left\|E_{f a r}^{C O M S O L}\right\|_{L^{2}(I)}},
$$

where $\|\cdot\|_{L^{2}(I)}$ is the usual $L^{2}$-norm over the angle interval I. The numerical analysis in this paper is made with such in- 
verse scattering applications in mind where only the intensity (amplitude) of the scattered far field is available, and it is therefore also relevant to define the relative $L^{2}$-error in the MAS far field amplitude,

$$
\varepsilon_{\text {far }}^{A B S}(I)=\frac{\left\|\left|E_{f a r}^{C O M S O L}\right|-\left|E_{f a r}^{M A S}\right|\right\|_{L^{2}(I)}}{\left\|E_{f a r}^{C O M S O L}\right\|_{L^{2}(I)}} .
$$

Since, for any complex $a$ and $b$, it holds that ||$a|-| b|| \leq|a-b|$, it is always the case that $\varepsilon_{f a r}^{A B S}(I) \leq \varepsilon_{f a r}(I)$. The relative boundary condition error at the nanowire cross section boundary $\sigma$ is defined for the electric and the magnetic fields as

$$
\begin{aligned}
\varepsilon_{\text {near }}^{E} & =\frac{\left\|\widehat{\mathbf{z}} \cdot\left(\mathbf{E}_{\sigma+}^{s}-\mathbf{E}_{\sigma-}^{t o t}+\mathbf{E}^{i}+\mathbf{E}^{r e f l}\right)\right\|_{L^{2}(\sigma)}}{\left\|\widehat{\mathbf{z}} \cdot\left(\mathbf{E}^{i}+\mathbf{E}^{r e f l}\right)\right\|_{L^{2}(\sigma)}}, \\
\varepsilon_{\text {near }}^{H} & =\frac{\left\|\widehat{\rho} \times\left(\mathbf{H}_{\sigma+}^{s}-\mathbf{H}_{\sigma-}^{\text {tot }}+\mathbf{H}^{i}+\mathbf{H}^{r e f l}\right)\right\|_{L^{2}(\sigma)}}{\left\|\widehat{\rho} \times\left(\mathbf{H}^{i}+\mathbf{H}^{r e f l}\right)\right\|_{L^{2}(\sigma)}},
\end{aligned}
$$

with the understanding that $\mathbf{E}_{\sigma-}^{\text {tot }}=0$ in the PEC case. Finally, we mention that, for practical reasons (the scattering problem geometry can not be included in a finite box in the COMSOL model,) all far-field quantities are here evaluated at a distance of $12.5 \lambda_{0}$ from the nanowire. Also, the MAS far-field results are obtained using the full expression for the Hankel function $H_{0}^{(2)}$, rather than an asymptotic one.

Figures 5 and 6 show the values of $\varepsilon_{\text {near }}^{E}, \varepsilon_{f a r}\left(\left[0^{\circ}, 90^{\circ}\right]\right)$ and $\varepsilon_{f a r}^{A B S}\left(\left[0^{\circ}, 90^{\circ}\right]\right)$ averaged over 11 PEC nanowires with radii $0.2 \lambda_{0}, 0.3 \lambda_{0}, \ldots, 1.2 \lambda_{0}$. The average relative errors are plotted as function of the number $N$ of auxiliary sources and testing points used in the MAS formulation. The averaging is done because we wish to use the present MAS forward model in an inversion scheme where the radius of the nanowire cross section is only known to be within a rather large interval, and we need to know the performance of the model over the whole interval. Also, it is certainly possible to allow a change (increase) in the number $N$ of auxiliary sources and testing points as the nanowire radius $r$ increases from $0.2 \lambda_{0}$ to $1.2 \lambda_{0}$, and thereby obtain better far-field results compared to a scheme with fixed $N$. However, we wish to use the MAS model in an inversion that employs an optimiser with a fixed number of optimisation variables, which is why $N$ is to be kept constant over the whole radius range.

The boundary condition error $\varepsilon_{\text {near }}^{E}$ is strictly decreasing as $N$ increases, while the relative far-field error $\varepsilon_{f a r}\left(\left[0^{\circ}, 90^{\circ}\right]\right.$ and the relative far-field amplitude error $\varepsilon_{f a r}^{A B S}\left(\left[0^{\circ}, 90^{\circ}\right]\right)$ seem to approach the finite levels of $6.86 \%$, and $4.43 \%$, respectively. These asymptotic error levels are nearly reached already for $N=15$, corresponding to mean $\varepsilon_{\text {near }}^{E} \approx 0.54 \%$. For the narrower far-field observation interval $\left[45^{\circ}, 90^{\circ}\right]$, we found that the errors $\varepsilon_{f a r}\left(\left[45^{\circ}, 90^{\circ}\right]\right)$ and $\varepsilon_{f a r}^{A B S}\left(\left[45^{\circ}, 90^{\circ}\right]\right)$ approach the values $1.55 \%$ and $1.16 \%$, respectively, for increasing $N$. In this case, the asymptotic error levels are nearly attained already for $N=22$, corresponding to mean $\varepsilon_{\text {near }}^{E} \approx 0.04 \%$. The far-field errors in the interval $\left[45^{\circ}, 90^{\circ}\right]$ are investigated because, in realistic particle-on-surface characterisation, the farfield measurements are usually available only in an angular range smaller than $\left[0^{\circ}, 90^{\circ}\right]$. The reason why the present MAS formulation is nonconvergent (the far-field error does not approach zero as $N$ approaches infinity) is the use of the Fresnel

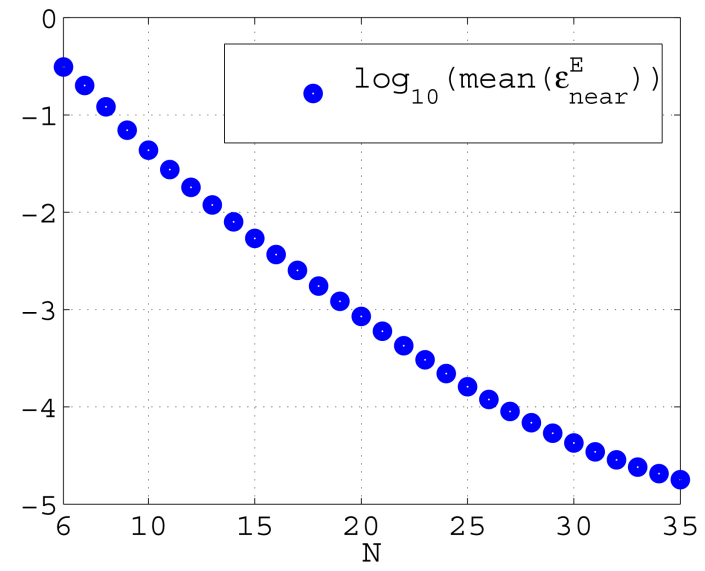

FIG. 5 The average relative boundary error as function of the number of auxiliary sources. (11 PEC nanowires with $r=0.2 \lambda_{0}, \ldots, 1.2 \lambda_{0}$ ).

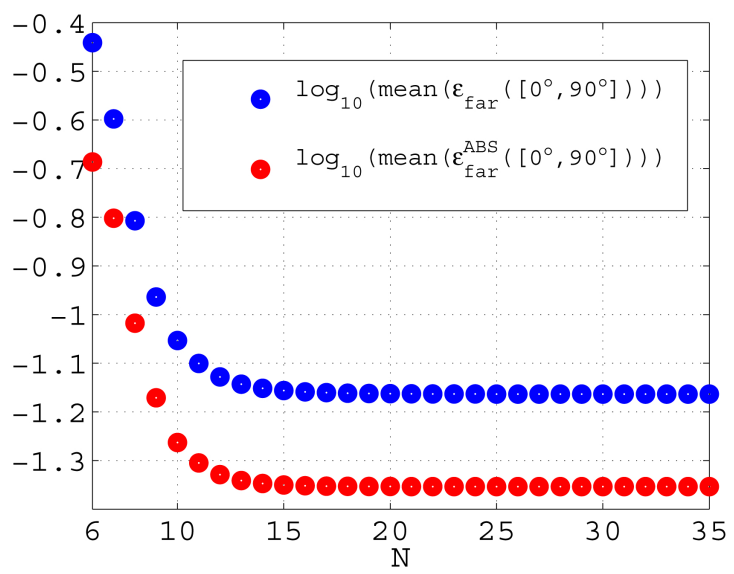

FIG. 6 The average relative far-field and far-field amplitude error as function of the number of auxiliary sources. Far field observed between $0^{\circ}$ and $90^{\circ}$. (11 PEC nanowires with $\left.r=0.2 \lambda_{0}, \ldots, 1.2 \lambda_{0}\right)$.

reflection approximation for the half-plane Green's function, described earlier.

The $l^{2}$-norm of the auxiliary source amplitude vector $\mathbf{C}=\left(C_{1}, \ldots, C_{N}\right)$ is shown in Figure 7 for 6 PEC nanowires as function of $N$. The MAS forward model presented here is intended for use in a Tikhonov-regularised optimisation, and it is therefore of interest to know the level of $\|\mathbf{C}\|_{l^{2}}$ obtained when solving the direct problem. It is seen that the norm $\|\mathbf{C}\|_{l^{2}}$ increases with the nanowire radius but decreases with $N$, for sufficiently large $N$. The average norm $\|\mathbf{C}\|_{l^{2}}$, taken over all 11 PEC nanowires, is approximately 2.01 and 1.66 at $N=15$ and $N=22$, respectively (the values of $N$ where the far-field error stabilises.)

Figures 8 and 9 show the average relative errors for the $\mathrm{Ag}$ nanowire as function of the number $N$ of auxiliary sources and testing points. Again, 11 nanowires are considered, with radii $0.2 \lambda_{0}, 0.3 \lambda_{0}, \ldots, 1.2 \lambda_{0}$. The average $l^{2}$-norm of the interior, exterior and total auxiliary source amplitude vectors $\mathbf{C}=\left(C_{1}, \ldots, C_{N}\right), \mathbf{D}=\left(D_{1}, \ldots, D_{N}\right)$ and $\left(C_{1}, \ldots, C_{N}, D_{1}, \ldots, D_{N}\right)$ is shown in Figure 10. Note that mean $\|\mathbf{C}\|_{l^{2}}$ is about three times smaller than mean $\|\mathbf{D}\|_{l^{2}}$ 


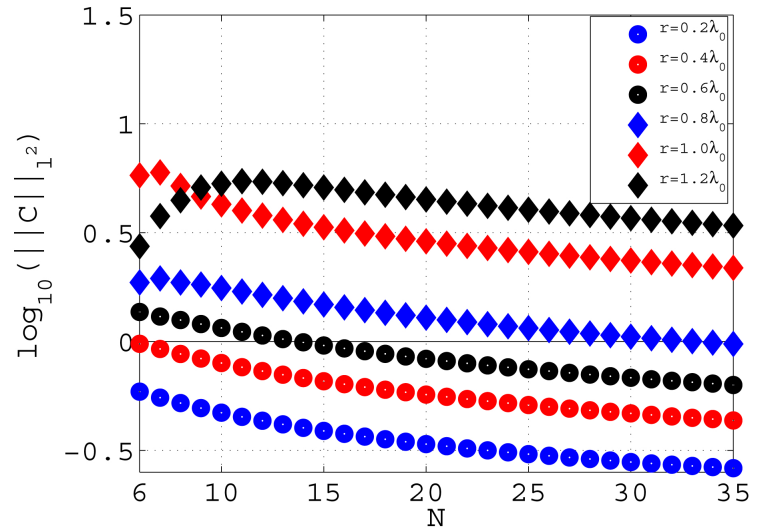

FIG. $7\|\mathbf{C}\|_{l^{2}}$ as function of the number $N$ of auxiliary sources and testing points: PEC nanowires.

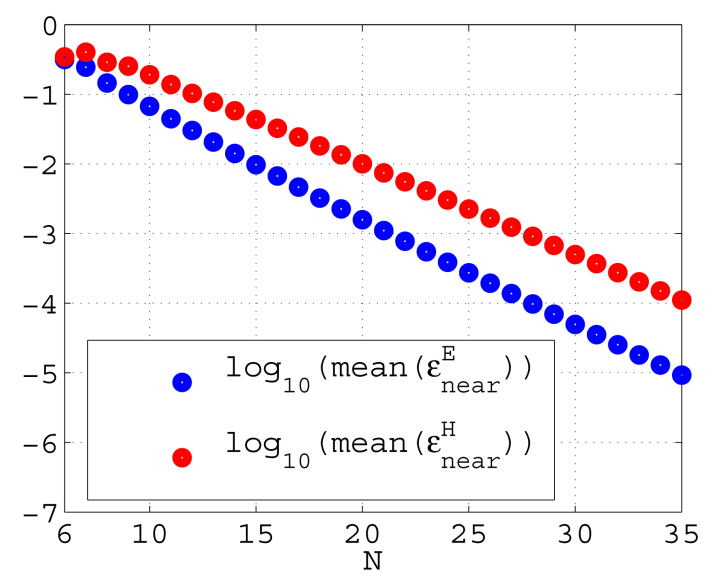

FIC. 8 The average relative electric field and magnetic field boundary error as function of the number of auxiliary sources. (11 Ag nanowires with $r=0.2 \lambda_{0}, \ldots, 1.2 \lambda_{0}$ ).

for large values of $N$. The boundary condition errors $\varepsilon_{\text {near }}^{E}$ and $\varepsilon_{\text {near }}^{H}$ for the tangential electric and tangential magnetic field, respectively, are both strictly decreasing with increasing $N$, although not at the same rate. The far-field errors $\varepsilon_{f a r}\left(\left[0^{\circ}, 90^{\circ}\right]\right)$ and $\varepsilon_{f a r}^{A B S}\left(\left[0^{\circ}, 90^{\circ}\right]\right)$ approach the levels $6.5 \%$ and $4.2 \%$, respectively, as $N$ increases. These asymptotic error levels are nearly reached at $N=25$, corresponding to mean $\varepsilon_{\text {near }}^{E} \approx 0.027 \%$ and mean $\varepsilon_{\text {near }}^{H} \approx 0.23 \%$. For the narrower far-field observation interval $\left[45^{\circ}, 90^{\circ}\right]$, we found that the errors $\varepsilon_{f a r}\left(\left[45^{\circ}, 90^{\circ}\right]\right)$ and $\varepsilon_{f a r}^{A B S}\left(\left[45^{\circ}, 90^{\circ}\right]\right)$ approach the values $1.65 \%$ and $1.27 \%$, respectively, for increasing $N$. These asymptotic error levels are nearly attained already for $N=23$, corresponding to mean $\varepsilon_{\text {near }}^{E} \approx 0.05 \%$ and mean $\varepsilon_{\text {near }}^{H} \approx 0.41 \%$. At $N=25$ and $N=23$, the average $l^{2}$-norm $\sqrt{\|\mathbf{C}\|_{l^{2}}^{2}+\|\mathbf{D}\|_{l^{2}}^{2}}$ of the total auxiliary source amplitude vector is approximately 2.33 and 2.42 , respectively.

Using the MAS formulation described here, with $N=15$ in the PEC case and $N=20$ in the penetrable case, the computation of the auxiliary source amplitude vector $\mathrm{C}$ (PEC case) or $(\mathbf{C}, \mathbf{D})$ (penetrable case) takes approximately $0.4 \mathrm{sec}$ and $1.6 \mathrm{sec}$, respectively. The computation is done in MATLAB ${ }^{\circledR}$ on a single $2.66 \mathrm{GHz}$ standard PC core, with $4 \mathrm{~Gb}$ of memory. The subsequent computation of the electric far field at 1600 points, used in the comparison with the COMSOL field in the

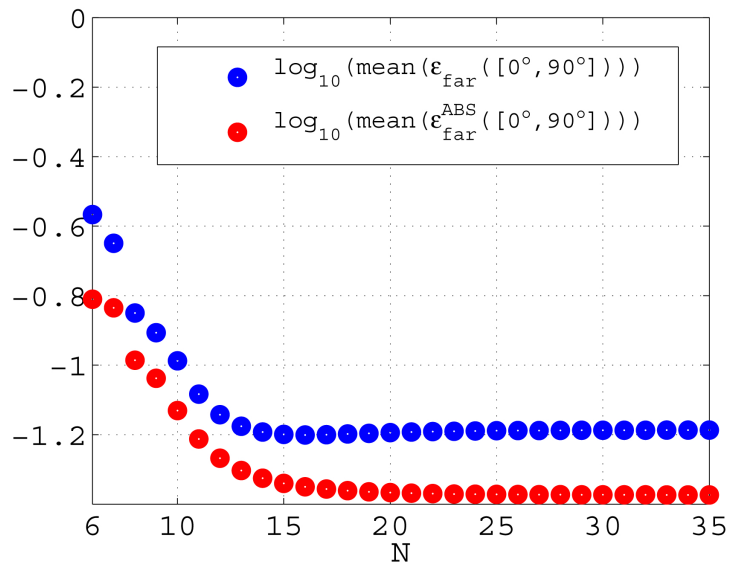

FIC. 9 The average relative far-field and far-field amplitude error as function of the number of auxiliary sources. Far field observed between $0^{\circ}$ and $90^{\circ}$. (11 $\mathrm{Ag}$ nanowires with $\left.r=0.2 \lambda_{0}, \ldots, 1.2 \lambda_{0}\right)$.

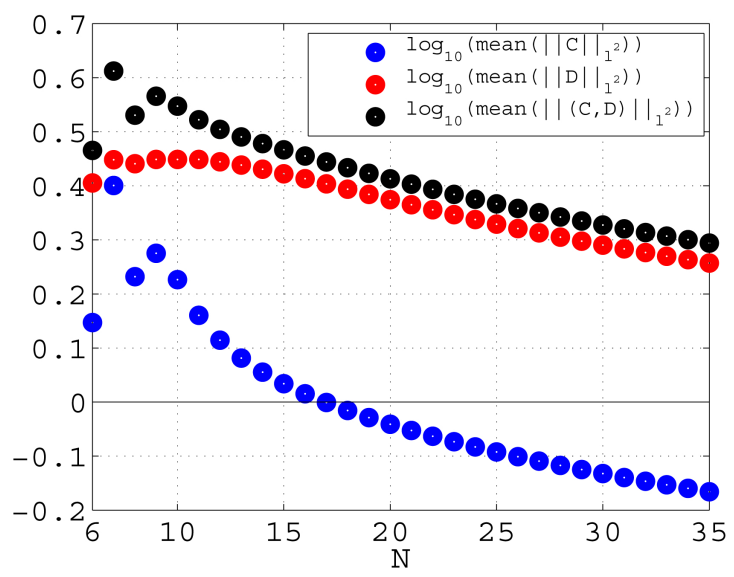

FIG. 10 Average $\|\mathbf{C}\|_{l^{2}},\|\mathbf{D}\|_{l^{2}}$ and $\sqrt{\|\mathbf{C}\|_{l^{2}}^{2}+\|\mathbf{D}\|_{l^{2}}^{2}}$ as function of the number $N$ of auxiliary sources and testing points. (11 Ag nanowires with $r=0.2 \lambda_{0}, \ldots, 1.2 \lambda_{0}$ ).

interval $\left[0^{\circ}, 90^{\circ}\right]$, takes approximately $18.4 \mathrm{sec}$ and $24.5 \mathrm{sec}$, respectively.

Finally, we conducted a study of the previously defined boundary condition error and far-field error as function of nanowire radius. In the PEC case, 15 auxiliary sources were used, and in the penetrable case $N=20$ was set. In both cases, the boundary condition error generally increased with increasing nanowire radius. For Ag nanowires, the magnetic field boundary condition error $\varepsilon_{\text {near }}^{H}$ was always larger than its electric counterpart. The boundary condition error for PEC nanowires was at most $1.54 \%$. The electric and magnetic boundary condition errors for the penetrable case were at most $0.41 \%$ and $1.71 \%$, respectively. The far-field error $\varepsilon_{f a r}\left(\left[0^{\circ}, 90^{\circ}\right]\right)$ and the far-field amplitude error $\varepsilon_{f a r}^{A B S}\left(\left[0^{\circ}, 90^{\circ}\right]\right)$ were at most $10.48 \%$ and $6.39 \%$, respectively, in the PEC case, and at most $8.95 \%$ and $5.35 \%$, respectively, in the penetrable case. For the narrower far-field observation interval $\left[45^{\circ}, 90^{\circ}\right]$, the far-field errors $\varepsilon_{f a r}\left(\left[45^{\circ}, 90^{\circ}\right]\right)$ and $\varepsilon_{f a r}^{A B S}\left(\left[45^{\circ}, 90^{\circ}\right]\right)$ were at most $3.51 \%$ and $3.08 \%$, respectively, in the PEC case, and at most $3.10 \%$ and $2.94 \%$, respectively, in the penetrable case. All these far-field errors seemed to be generally decreasing with increasing radius $r$, which can be explained by the fact 
that with the larger scatterers some of the auxiliary sources are positioned farther away from the air-silicon substrate, and hence are better approximated by the Fresnel reflection.

\section{CONCLUSION AND FURTHER WORK}

An efficient model for scattering by PEC and penetrable nanowires on dielectric substrates was formulated in terms of the Method of Auxiliary Sources (MAS). The accuracy and efficiency of the model were investigated parametrically using families of concrete scattering problems. In the investigation of the accuracy of the model, numerical solutions obtained using COMSOL Multiphysics were used as reference. Good model parameters were found for future use in an inversion scheme. In particular, for PEC nanowires with cross-section radius between $0.2 \lambda_{0}$ and $1.2 \lambda_{0}$ and placed on a silicon substrate, a good choice of the number of auxiliary sources and testing points to be employed in our implementation seemed to be approximately 15 . For silver (Ag) nanowires on silicon, a good choice of this number seemed to be approximately 20. With these choices, the relative far-field error and far-field amplitude error were less than $10.5 \%$ and $6.4 \%$, respectively, in the PEC case, and less than $9 \%$ and $5.4 \%$, respectively, in the penetrable $(\mathrm{Ag})$ case. These errors were smaller when the range of the far-field observation angle was reduced from the full $\left[0^{\circ}, 90^{\circ}\right]$ to $\left[45^{\circ}, 90^{\circ}\right]$.

The MAS formulation required a total of approximately $19 \mathrm{sec}$ and $26 \mathrm{sec}$ for the computation of the far field at 1600 observation points in the PEC case and in the penetrable case, respectively. The formulation could be sped up further by using the asymptotic expression for the Hankel function $H_{0}^{(2)}$ in the far field. Also, the presented MAS formulation is - at least in principle - readily extendible to scattering by arbitrarily shaped, two- or three-dimensional PEC and penetrable particles on substrates. For two-dimensional scattering problems with TM-polarised fields, magnetic instead of electric line currents should be used for the auxiliary sources. If the angle of incidence is greater than zero, the Fresnel reflection coefficients for the TE and the TM polarisations are different, and this should also be taken into account. Clearly, care should be taken that the boundary conditions valid at the particle surface are correctly implemented for the given polarisation. Finally, in the three-dimensional case, one good choice of sources could be (crossed) electric Hertzian dipoles.

\section{ACKNOWLEDGEMENTS}

We acknowledge the financial support from Deutsche Forschungsgemeinschaft (DFG), Grant No. WR 22/36-1.

\section{References}

[1] N. Agersnap, P.-E. Hansen, J. C. Petersen, J. Garnaes, N. Destouches, and 0. Parriaux, "Critical dimension metrology using optical diffraction microscopy" Proc. SPIE 5965, 68-78 (2005).

[2] J. Garnaes, P.-E. Hansen, N. Agersnap, I. Davi, J. C. Petersen, A. Kühle, J. Holm, and L. H. Christensen, "Determination of sub- micrometer high aspect ratio grating profiles" Proc. SPIE 5878, 1-9 (2005).

[3] J. Garnaes, P.-E. Hansen, N. Agersnap, J. Holm, F. Borsetto, and A. Kühle, "Profiles of high aspect ratio grating determined by optical diffraction microscopy and atomic force microscopy" Appl. Opt. 45, 3201-3212 (2006).

[4] P.-E. Hansen, and L. Nielsen, “Combined optimization and hybrid scalar-vector diffraction method for grating topography parameters determination" Mater. Sci. Eng. B-Adv. 165, 165-168 (2009).

[5] M. Karamehmedović, M.-P. Sørensen, P.-E. Hansen, and A. V. Lavrinenko, "Application of the method of auxiliary sources to a defectdetection inverse problem of optical diffraction microscopy" J. Eur. Opt. Soc. Rapid Publ. 5, 10021 (2010).

[6] A. Kirsch, and R. Kress, "On an integral equation of the first kind in inverse acoustic scattering" Internat. Schriftenreihe Numer. Math. 77, 93-102 (1986).

[7] D. Colton, and R. Kress, Inverse acoustic and electromagnetic scattering theory (Springer-Verlag, Berlin, 1998).

[8] V. D. Kupradze, "On the approximate solution of problems of mathematical physics" Russ. Math. Surv. 22, 59-107 (1967).

[9] G. Fairweather, and A. Karageorghis, "The method of fundamental solutions for elliptic boundary value problems" Adv. Comput. Math. 9, 69-95 (1998).

[10] G. Fairweather, A. Karageorghis, and P. A. Martin, "The method of fundamental solutions for scattering and radiation problems" Eng. Anal. Bound. Elem. 27, 759-769 (2003).

[11] A. Doicu, Y. A. Eremin, and T. Wriedt, Acoustic at Electromagnetic Scattering Analysis Using Discrete Sources (Academic Press, New York, 2000).

[12] T. Wriedt, Generalized multipole techniques for electromagnetic and light scattering (Elsevier, Amsterdam, 1999).

[13] D. I. Kaklamani, and H. T. Anastassiu, "Aspects of the method of auxiliary sources (MAS) in computational electromagnetics" IEEE Antennas Propag. M. 44, 48-64 (2002).

[14] Y. Leviatan, and A. Boag, "Analysis of electromagnetic scattering from dielectric cylinders using a multifilament current model" IEEE T. Antenn. Propag. AP-35, 1119-1127 (1987).

[15] J. Tal, and Y. Leviatan, "Inverse scattering analysis for perfectly conducting cylinders using a multifilament current model" Inverse Probl. 6, 1065-1074 (1990).

[16] C.-Y. Lin, and Y.-W. Kiang, "Inverse scattering for conductors by the equivalent source method" IEEE T. Antenn. Propag. 44, 310-316 (1996).

[17] F. Obelleiro, L. Landesa, J. L. Rodríguez, and M. R. Pino, “Fast twodimensional reconstruction of impenetrable objects using multipolar equivalent sources" IEEE T. Magn. 35, 1570-1573 (1999).

[18] C. J. S. Alves, and N. F. M. Martins, "The direct method of fundamental solutions and the inverse Kirsch-Kress method for the reconstruction of elastic inclusions or cavities" J. Integral Equat. Appl. 21, 153-178 (2009).

[19] E. Eremina, Y. Eremin, and T. Wriedt, "Discrete sources method for simulation of resonance spectra of nonspherical nanoparticles on a plane surface" Opt. Commun. 246, 405-413 (2005).

[20] E. Eremina, Y. Eremin, and T. Wriedt, "Analysis of the light scattering properties of a gold nanorod on a plane surface via discrete sources method" Opt. Commun. 273, 278-285 (2007).

[21] COMSOL Multiphysics demonstration CD-ROM can be requested at http://www.comsol.com. 
[22] W. B. J. Zimmerman, Multiphysics Modelling with Finite Element Methods (World Scientific, Singapore, 2006).
[23] E. D. Palik, Handbook of optical constants of solids (Academic Press, Boston, 1985). 